\title{
Induction gemcitabine-based chemotherapy and neoadjuvant stereotactic body radiation therapy achieve high margin-negative resection rates for borderline resectable pancreatic cancer
}

\author{
Michael D. Chuong • Gregory M. Springett • \\ Jill Weber • Jason Klapman • Shivakumar Vignesh • \\ Pamela J. Hodul • Mokenge P. Malafa • \\ Susan Leuthold • Sarah E. Hoffe • Ravi Shridhar
}

Received: 19 April 2012 / Accepted: 15 May 2012 / Published online: 30 May 2012

(C) Springer-Verlag 2012

\begin{abstract}
Objectives Patients with borderline resectable pancreatic cancer (BRPC) have a higher probability of undergoing margin-negative resection after completing neoadjuvant therapy. Here, we describe a novel neoadjuvant approach using induction chemotherapy followed by stereotactic body radiation therapy (SBRT) for patients with BRPC.

Methods This analysis included patients with nonmetastatic BRPC treated with neoadjuvant gemcitabine-based chemotherapy and five-fraction SBRT. Chemotherapy consisted of 3 cycles of Gemzar, Taxotere, and Xeloda. Patients were restaged to determine resectability, and nonmetastatic resectable patients underwent surgical resection.

Results Thirty patients completed neoadjuvant treatment and were offered surgical exploration. Seventeen patients (56.7 \%) reported no acute adverse effects during SBRT. No grade 3 or higher toxicity was observed from SBRT. Twenty-nine patients $(96.7 \%)$ underwent exploration. Twenty-one (95.6\%) of those who underwent pancreatic tumor resection achieved negative margins, with none requiring vessel resection. One (3.3\%) patient was resected
\end{abstract}

M. D. Chuong $(\bowtie) \cdot S$. Leuthold $\cdot$ S. E. Hoffe $\cdot$ R. Shridhar

Department of Radiation Oncology, H. Lee Moffitt Cancer Center

\& Research Institute,

12902 Magnolia Drive,

Tampa, FL 33612, USA

e-mail: michael.chuong@moffitt.org

G. M. Springett $\cdot$ J. Weber $\cdot$ J. Klapman $\cdot$ S. Vignesh $\cdot$ P. J. Hodul $•$

M. P. Malafa

Department of Gastrointestinal Oncology,

H. Lee Moffitt Cancer Center,

Tampa, FL, USA with microscopic positive margins. Median follow-up was 15.6 months (range, 6.3-26.1 months). Median and 1-year overall survival was 20 months and $91 \%$, respectively. Median and 1-year progression-free survival was 14.9 months and $61 \%$, respectively.

Conclusion SBRT-based neoadjuvant therapy for BRPC is well tolerated and can result in a high rate of marginnegative tumor resection.

Keywords Stereotactic body radiation therapy $\cdot$ Borderline resectable pancreatic cancer - GTX

\section{Introduction}

Pancreatic cancer is the fourth most common cause of cancer death in the USA [1]. While surgical resection remains the only chance for cure, the majority of patients are not surgical candidates at initial presentation due to either locally advanced or distant metastatic disease. Of the $20 \%$ that are resectable, the 5-year estimated survival remains quite poor at approximately $20 \%$ [2].

Approximately one third of patients presenting with locally advanced pancreatic cancer (LAPC) are classified as being borderline resectable. While variations on the definition of borderline resectable pancreatic cancer (BRPC) exist, these patients typically have abutment, but not complete encasement, of nearby vasculature $[3,4]$. Such abutment significantly lowers the probability of achieving a marginnegative resection (R0), and consequently the likelihood of cure. Patients who undergo resection with positive surgical 
margins (R1 or R2) have outcomes similar to those who did not undergo resection [5-7]. Thus, the importance of achieving an R0 resection cannot be understated.

Neoadjuvant chemoradiation has been shown to have several theoretical and practical benefits for patients with BRPC. First, data from our institution as well as from others have shown that neoadjuvant chemoradiation for BRPC can significantly increase the likelihood of undergoing an R0 resection, which may improve overall survival [8-11]. Of note, these studies used radiotherapy techniques with standard fractionation over approximately 5 weeks. Second, exposure of the tumor to chemotherapeutic agents prior to resection allows for the sensitivity of the tumor to those agents to be assessed. If the tumor responds, then the same therapy may be employed if the patient has a recurrence. Third, intensive multiagent multimodality treatment programs may be better tolerated by patients preoperatively versus postoperatively. Patients who experience major complications after surgery may require very prolonged recovery times during which chemotherapy and radiation might not be given. Fourth, radiosensitizing oxygen and chemotherapy is more readily delivered within the tumor tissue prior to surgery, making radiation therapy more effective. Surgery may potentially disrupt blood flow to the operative bed and might limit the effectiveness of postoperative radiation. Finally, most successfully resected patients nevertheless recur, which is de facto evidence that micrometastasis is present at an early stage of the disease. Neoadjuvant therapy potentially allows for the early control of occult micrometastasis.

Just as the transition from 5-fluorouracil- to gemcitabinebased chemotherapy regimens has emerged, the evolution of radiation therapy (RT) techniques has also resulted in improvements in patient outcomes. When compared to 3D conformal RT (3D-CRT), intensity-modulated RT (IMRT) is able to produce more tightly conformal isodose distributions while better minimizing dose to nearby critical normal structures, decreasing acute and late complications [12-14]. A technique known as stereotactic body radiation therapy (SBRT) has been utilized in various extracranial sites, with the most experience being in early stage nonsmall cell lung cancer $[15,16]$.

SBRT allows very high ablative doses of radiation to be precisely given to small target volumes over several days compared to standard treatment that is commonly delivered over 5 weeks. While SBRT has been performed in the treatment of abdominal malignancies including in LAPC $[17,18]$, its use has not been reported in the treatment of BRPC. Previous SBRT studies in the setting of LAPC have demonstrated technical feasibility, high rates of local control, and minimal toxicity [19-24]. Based on these data, we developed a defined treatment pathway at our institution for the diagnosis, staging, and management of BRPC that involves the use of neoadjuvant chemotherapy followed by
SBRT. We now report on the outcomes of BRPC patients treated at our institution that completed treatment with induction gemcitabine-based chemotherapy and SBRT.

\section{Methods}

Patient details

After IRB approval, the medical records of non-metastatic BRPC patients who completed induction gemcitabine-based chemotherapy followed by SBRT at our institution were reviewed. Since a standard approach to treating BRPC does not exist, our institution developed a pathway for the management of BRPC that includes induction GTX (Gemzar, Taxotere, Xeloda) chemotherapy followed by SBRT and restaging. Published data have shown both GTX chemotherapy in combination with RT to be effective and well tolerated in locally advanced pancreatic cancer [8, 25]. All patients were treated with curative intent between December 2009 and July 2011. No patient had prior chemotherapy or abdominal radiation therapy.

\section{Staging}

The initial evaluation included a detailed history and physical examination and routine blood chemistries. We obtained a multi-detector thin-section pancreatic protocol computed tomography (CT) scan, endoscopic ultrasound (EUS), and positron emission tomography (PET) scan for each patient in accordance with our Multidisciplinary Pancreatic Cancer Clinic staging algorithm. Although PET scan is not considered to be standard for pancreatic cancer staging, our center is a participant in the National Oncologic PET Registry, which allows us to evaluate this imaging modality in pancreatic cancer. Our institutional data have shown that metabolic imaging can change management in $11 \%$ of pancreatic cancer cases [26]. The diagnosis of BRPC was confirmed at our GI Multidisciplinary Tumor Board prior to each patient beginning treatment by a surgeon and radiologist who both specialized in GI malignancies.

Definition of borderline resectable pancreatic cancer

Borderline resectable pancreatic cancer was diagnosed based on EUS and CT findings. In the event of a discrepancy between imaging studies, the highest stage finding was used. The following criteria were used when defining borderline resectable disease: $(1) \leq 180^{\circ}$ circumferential tumor abutment with the superior mesenteric vein (SMV), portal vein (PV), or superior mesenteric artery (SMA); (2) short segment encasement (approximately $1.5 \mathrm{~cm}$ ) of the $\mathrm{PV} /$ SMV amenable to partial vein resection and reconstruction; 
or (3) gastroduodenal artery encasement up to the origin of the hepatic artery. Tumors of the head or body with involvement of both the PV/SMV and SMA that would require resection and reconstruction of both arterial and venous systems were classified as unresectable. Patients with encasement of the SMA, celiac artery, aorta, or inferior vena cava (IVC) were also classified as unresectable.

\section{Chemotherapy}

All patients received gemcitabine-based chemotherapy followed by SBRT. Each 21-day cycle of GTX was administered as follows: gemcitabine (Eli Lilly) $750 \mathrm{mg} / \mathrm{m}^{2}$ on days 4 and 11, docetaxel (Sanofi Aventis) $30 \mathrm{mg} / \mathrm{m}^{2}$ on days 4 and 11 , and capecitabine (Roche) $750 \mathrm{mg} / \mathrm{m}^{2}$ BID on days 1-14. Patients completed the prescribed 3 cycles with dose reductions as recommended by the treating medical oncologist (GS) for chemotherapy-related adverse effects.

\section{Radiation treatment planning and delivery}

Endoscopically placed Visicoil (Core Oncology, Santa Barbara, CA) fiducial markers were placed in the tumor prior to simulation to optimize target delineation, both during treatment planning as well as during daily setup verification after induction chemotherapy. One to three fiducial markers $(0.35 \times 1 \mathrm{~cm})$ were implanted into the peritumoral tissue. Variability in the number of markers resulted from technical difficulties during placement. Prior to CT simulation, the extent of tumor motion from respiration was determined under fluoroscopy using the fiducial markers. Motion management strategies were personalized to each patient. Patients were assessed to determine whether respiratory-gated SBRT with delivery in maximum exhalation phase vs. abdominal compression vs. free breathing with larger margins and an internal target volume (ITV) approach would be utilized. The majority of patients in this series were treated with gated SBRT using 3D conformal techniques. Those patients who received IMRT all had verified motion with proper management strategies less than $1 \mathrm{~cm}$.

CT simulation was performed with the patient supine and arms overhead in a BodyFIX (Elekta, Stockholm, Sweden) immobilization cradle. Oral gastrografin was administered $30 \mathrm{~min}$ prior to immobilization. A free breathing scan from the carina to iliac crest in 3-mm increments using IV and oral contrast was first done to set the isocenter. A 4D CT scan was then performed to quantify the degree of tumor movement with respiration.

To account for tumor motion during respiration, we used several techniques including abdominal compression. This minimized diaphragmatic excursion and, hence, primarily superior-to-inferior tumor motion. Respiratory gating has more recently become available at our institution, which allowed us to deliver dose to the target only during a particular phase of the respiratory cycle, specifically during end expiration when tumor motion was typically the least variable. The respiratory cycle was recorded using an abdominal bellows strap placed at the inferior costal margin. The free breathing respiratory trace was divided into ten phases, of which the $50 \%$ phase (usually maximum end expiration) was typically selected for treatment planning. The $50 \%$ phase was fused to the 40 and $60 \%$ phases, which defined the gating window during which the photon beam was turned on. Diaphragmatic excursion, and indirectly tumor position, was tracked during treatment using an infrared reflector placed along the right infracostal border.

After CT simulation, the images were transferred to a Pinnacle workstation where the target volumes were contoured by an attending radiation oncologist (SEH, RS). The intent was to use dose painting to selectively deliver a higher dose to the portion of the tumor abutting the vasculature. To achieve this, two planning target volumes (PTVs) were created, one to encompass the entire extent of radiographic disease and a second to encompass that portion of tumor adjacent to the vasculature resulting in the borderline designation. Elective nodes were not included to limit normal tissue toxicity. For the gating patients, tumor was contoured on the 40,50 , and $60 \%$ phases to create an exhale ITV, accounting for motion in the gating window. For the abdominal compression patients, tumor was contoured on all phases to create an ITV. A 3-5-mm PTV expansion was intended to account for daily setup error.

SBRT began 1 week after completion of chemotherapy on a Varian Trilogy unit using $6-15-\mathrm{MeV}$ photons. Eight patients $(26.7 \%)$ were treated using SmartArc (Philips, The Netherlands) with abdominal compression. Twenty-one patients $(70 \%)$ were gated. One $(3.3 \%)$ was treated in free breathing after not tolerating the weight belt, prior to the availability of respiratory gating at our center. Fiducial marker position was verified prior to treatment using cone beam CT and fluoroscopy.

Five-fraction SBRT was delivered with the intent to deliver up to $30-40$ Gy to the tumor-vessel interface and up to 2530 Gy to the remainder of the gross disease. Dosing was limited by the ability to meet normal tissue constraints, particularly with respect to the small bowel. Normal tissue constraints are as follows: kidney (mean $<10 \mathrm{~Gy}$ ), spinal cord (maximum $20 \mathrm{~Gy}$ ), liver (mean $<10 \mathrm{~Gy}$ ), stomach, duodenum, and bowel ( $1 \mathrm{cc}<35 \mathrm{~Gy}, 5 \mathrm{cc}<30 \mathrm{~Gy}$, mean $<20 \mathrm{~Gy})$.

Surgical resection

Restaging PET and pancreas protocol CT scans were obtained approximately 3-4 weeks after completion of SBRT and were reviewed at our GI Tumor Board for consideration of exploratory laparotomy and possible surgical resection. Those patients with tumors involving the pancreatic head underwent 
pancreaticoduodenectomy, while those with tumors involving the body underwent distal pancreatectomy and splenectomy. The operating surgeon and pathologist determined whether an $\mathrm{R} 0$ or R1/2 resection was achieved. Vascular surgeons were available in the event that any patient required a vessel resection.

Follow-up

Adverse effects were assessed according to the National Cancer Institute Common Terminology Criteria for Adverse Events, version 4.0 [2]. Acute effects were considered to be those that occurred within 90 days of SBRT completion, and late effects were those that occurred more than 90 days after SBRT completion. Patients were seen in clinic every 36 months postoperatively or after SBRT completion if they remained unresectable.

\section{Results}

Thirty patients were identified who met inclusion criteria. Table 1 summarizes patient and tumor characteristics. The median age was 64 years (range, 44-77 years). Tumors were most commonly located in the head of the pancreas (90\%). One patient had biopsy-proven adenosquamous carcinoma, while all other tumors were adenocarcinoma. Blood vessels clinically involved by tumor based on staging workup most commonly involved the SMV alone (36.7\%), PV alone (26.7\%), or PV/SMV confluence (20\%). Multiple vessel involvement was diagnosed in eight patients $(26.7 \%)$. One patient $(3.3 \%)$ had clinical T4 disease, and 18 patients (60\%) had clinical node-positive disease by EUS staging. Twenty-seven of 30 (89 \%) patients received GTX chemotherapy at our institution, and the rest received gemcitabinebased chemotherapy at our affiliated community practices. One patient had gemcitabine for 1 cycle followed by 2 cycles of GTX, one patient was treated with gemcitabine alone, and one patient received erlotinib with the GTX regimen. The median planning target volume encompassing the entire tumor versus the tumor-vessel interface alone was $116.5 \mathrm{~cm}^{3}$ (range, $72.0-255.4 \mathrm{~cm}^{3}$ ) and $17.5 \mathrm{~cm}^{3}$ (range, $6.9-75.1 \mathrm{~cm}^{3}$ ), respectively. The entire tumor volume received a median of 5 Gy per fraction (range, 5-6 Gy) for a total median dose of 25 Gy (range, 25-30 Gy). The tumor volume abutting vasculature received a total median dose of 32.8 Gy (range, 30-40 Gy) in 6-8 Gy fractions (median, 6.6 Gy). A typical BRPC case showing abutment of SMV, isodose distribution of dose-painted SBRT, and response to treatment is demonstrated in Fig. 1.

The median follow-up was 15.6 months (range, 6.326.1 months). Treatment was well tolerated. Seventeen patients $(56.7 \%)$ reported no acute adverse effects during
SBRT. There was no reported acute grade $3+$ toxicity in any patient. Seven $(23.3 \%)$ reported grade 1 fatigue that selfresolved shortly after finishing treatment, two $(6.6 \%)$ had grade 2 diarrhea, four (13.3\%) had grade 2 abdominal pain, and one $(3.3 \%)$ had grade 2 nausea. The adverse events are summarized in Table 2. While long-term follow-up is limited, there were no reported late adverse effects.

Table 1 Patient and treatment characteristics

\begin{tabular}{|c|c|c|}
\hline Characteristic & $N$ (range) & $\%$ \\
\hline Patients & 30 & \\
\hline Median age (years) & $64(44-77)$ & \\
\hline \multicolumn{3}{|l|}{ Clinical T stage } \\
\hline $\mathrm{T} 3$ & 29 & 96.7 \\
\hline $\mathrm{T} 4$ & 1 & 3.3 \\
\hline \multicolumn{3}{|l|}{ Clinical N stage } \\
\hline N0 & 12 & 40 \\
\hline N1 & 18 & 60 \\
\hline \multicolumn{3}{|l|}{ Tumor location } \\
\hline Head & 27 & 90 \\
\hline Body & 3 & 10 \\
\hline \multicolumn{3}{|l|}{ Involved vessel(s) } \\
\hline SMV alone & 11 & 36.7 \\
\hline PV alone & 8 & 26.7 \\
\hline $\mathrm{SMV} / \mathrm{PV}$ & 6 & 20 \\
\hline SMV/SMA & 1 & 3.3 \\
\hline $\mathrm{SMV} / \mathrm{SV}$ & 1 & 3.3 \\
\hline SMA alone & 1 & 3.3 \\
\hline SA & 1 & 3.3 \\
\hline IVC & 1 & 3.3 \\
\hline \multicolumn{3}{|l|}{ Chemotherapy } \\
\hline GTX alone & 27 & 27 \\
\hline Gemcitabine then GTX & 1 & 3.3 \\
\hline Gemcitabine alone & 1 & 3.3 \\
\hline Erlotinib then GTX & 1 & 3.3 \\
\hline \multicolumn{3}{|l|}{ Median PTV $\left(\mathrm{cm}^{3}\right)$} \\
\hline Entire tumor & $116.5(72-255.4)$ & \\
\hline Region of vessel abutment & $17.5(6.9-75.1)$ & \\
\hline \multicolumn{3}{|l|}{ Median SBRT dose } \\
\hline Entire tumor & 25 Gy $(25-30)$ & \\
\hline Region of vessel abutment & 32.8 Gy $(30-40)$ & \\
\hline \multicolumn{3}{|l|}{ SBRT technique } \\
\hline 3D-CRT & 1 & 3.3 \\
\hline $3 \mathrm{D}$ gating & 21 & 70 \\
\hline IMRT & 8 & 26.7 \\
\hline
\end{tabular}

$S M A$ superior mesenteric artery; $S M V$ superior mesenteric vein; $P V$ portal vein; IVC inferior vena cava; $S A$ splenic artery; GTX Gemzar, Taxotere, Xeloda; $S B R T$ stereotactic body radiation therapy; $3 D-C R T$ $3 \mathrm{D}$ conformal radiation therapy; IMRT intensity-modulated radiation therapy; $P T V$ planning target volume 

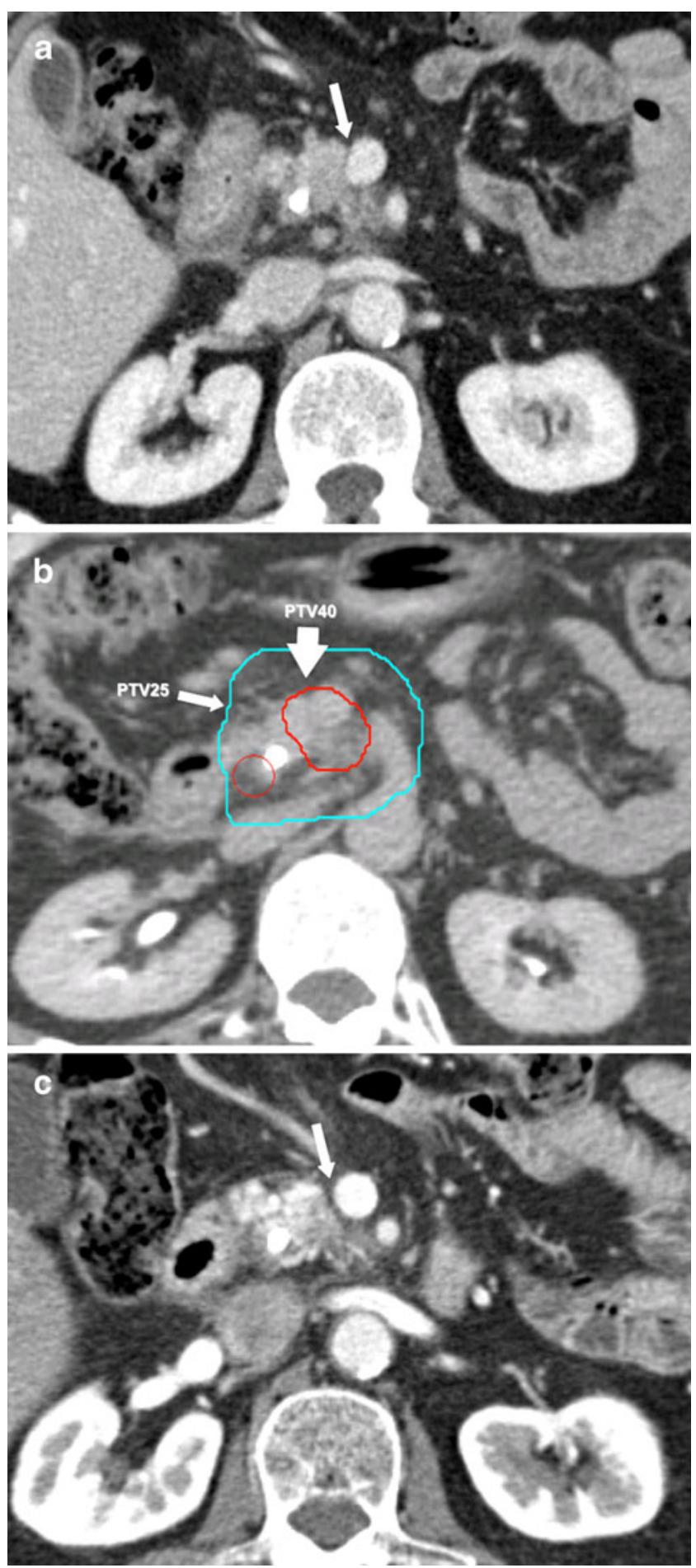

Fig. 1 a Pancreatic protocol CT of the abdomen (venous phase) illustrating abutment of pancreatic tumor to the SMV (arrow). b Isodose distribution of dose-painted SBRT treating the entire pancreatic tumor to $25 \mathrm{~Gy}$ and the area of vessel abutment (thick arrow) to 40 Gy in five fractions. c Radiographic response 4 weeks after SBRT showing a fat plane that developed between tumor and SMV (arrow) after neoadjuvant therapy

Table 3 describes the surgical outcomes. All 30 patients were considered to have had at least a partial radiographic response to neoadjuvant therapy based on visualization of a potential plane for resection between the tumor and nearby vasculature. As a result, all patients were recommended to undergo at least surgical exploration and then ideally pancreatic resection based on the findings at the time of exploration. Twenty-nine patients $(96.7 \%)$ underwent surgical exploration. Nine patients $(30 \%)$ did not undergo surgical resection. One (3.3\%) developed cardiovascular instability after preoperative cardiac clearance, five $(16.7 \%)$ developed distant metastatic disease not detected during initial staging but detected intraoperatively, two $(6.7 \%)$ had unresectable disease, and one (3.3\%) declined surgery although was medically fit. Unresectable and metastatic patients were recommended to continue management with chemotherapy. Twenty-one patients $(70 \%)$ were deemed resectable and underwent either pancreaticoduodenectomy (60\%) or distal pancreatectomy (10\%). Segmental resection of the SMA, PV, or SMV/PV confluence was not required in any patient. Vessel repair was required in two patients. Twenty resected patients achieved negative margins (95.2\%). Two patients $(6.7 \%)$ achieved a pathologic complete response, and one patient (3.3\%) had a near complete response with $<1 \mathrm{~mm}$ of residual disease. A pancreaticoduodenectomy was performed in one patient who was thought to have achieved negative margins intraoperatively, although ultimately was found to have a microscopically positive margin $(4.8 \%)$. Tumors were well differentiated $(14.3 \%)$, moderately differentiated $(28.6 \%)$, poorly differentiated (52.4\%), and undifferentiated (4.8\%). A median of 19 lymph nodes was resected (range, 1-46); 5 $(23.8 \%)$ resected patients were node-positive and 18 (76.2 \%) were node-negative. The median length of hospitalization was 10 days (range, 7-14 days). Postoperative complications were minimal; three patients developed wound infection, three had a pancreatic leak, and one had an ileus.

Figure 2 illustrates survival outcomes of BRPC patients who completed neoadjuvant therapy. Median and 1-year overall survival (OS) was 20 months and $91 \%$, respectively. Median and 1-year progression-free survival (PFS) was 14.9 months and $61 \%$, respectively.

Table 2 Worst acute and late adverse effects after completing induction chemotherapy and SBRT

\begin{tabular}{lcccc}
\hline & \multicolumn{3}{l}{ Grade (\%) } & \\
\cline { 2 - 5 } & 0 & 1 & 2 & $3+$ \\
\hline Acute & $17(56.7)$ & $7(23.3)$ & $7(23.3)$ & 0 \\
Fatigue & $6(20)$ & 0 & 0 \\
Nausea & $2(6.7)$ & $1(3.3)$ & 0 \\
Diarrhea & 0 & $2(6.7)$ & 0 \\
Abdominal pain & & 0 & $4(13.3)$ & 0 \\
Late & $30(100)$ & 0 & 0 & 0 \\
\hline
\end{tabular}


Table 3 Surgical characteristics after completing induction chemotherapy and SBRT

\begin{tabular}{|c|c|c|}
\hline & $N$ (range) & $\%$ \\
\hline Surgical exploration & 29 & 96.7 \\
\hline Tumor resection & 21 & 70 \\
\hline Pancreaticoduodenectomy & 18 & 60 \\
\hline Distal pancreatectomy & 3 & 10 \\
\hline No tumor resection & 9 & 30 \\
\hline Unresectable & 2 & 6.7 \\
\hline Distant metastasis & 5 & 16.7 \\
\hline Cardiac dysfunction & 1 & 3.3 \\
\hline Patient declined & 1 & 3.3 \\
\hline \multicolumn{3}{|l|}{ Surgical margin } \\
\hline Negative (R0) & 20 & 95.2 \\
\hline Microscopically positive (R1) & 1 & 4.8 \\
\hline Grossly positive (R2) & 0 & 0 \\
\hline \multicolumn{3}{|l|}{ Lymph nodes } \\
\hline pN0 patients & 16 & 76.2 \\
\hline pN1 patients & 5 & 23.8 \\
\hline Median total nodes resected & $19(1-46)$ & \\
\hline Median positive nodes resected & $3(1-5)$ & \\
\hline \multicolumn{3}{|l|}{ Tumor grade } \\
\hline Well differentiated & 3 & 14.3 \\
\hline Moderately differentiated & 6 & 28.6 \\
\hline Poorly differentiated & 11 & 52.4 \\
\hline Undifferentiated & 1 & 4.8 \\
\hline
\end{tabular}

\section{Discussion}

The management of BRPC continues to evolve. The Gastrointestinal Tumor Study Group trials established chemotherapy and radiation therapy as being superior to either modality alone $[27,28]$. This combined modality strategy has been adopted for the neoadjuvant treatment of borderline resectable pancreatic cancer, with the available data illustrating that it can markedly increase the likelihood of an $\mathrm{R} 0$ resection [8-10]. What is less certain is the optimal chemotherapy and radiation therapy regimen.

We previously published our initial results treating BRPC with induction GTX followed by radiation therapy [8]. The rationale behind using induction GTX was detailed in our initial study. It is important to note that our initial experience was performed using standard fractionation RT over several weeks. However, we have since transitioned to treating all BRPC patients with SBRT over 5 days based on the accruing SBRT data for LAPC. We now report the first series of BRPC treated with induction GTX followed by SBRT.

Our data show that SBRT is not only well tolerated, but also that dose painting the area of vessel abutment to a maximum of 8 Gy over five fractions effectively facilitated margin-negative pancreatic tumor resection without the
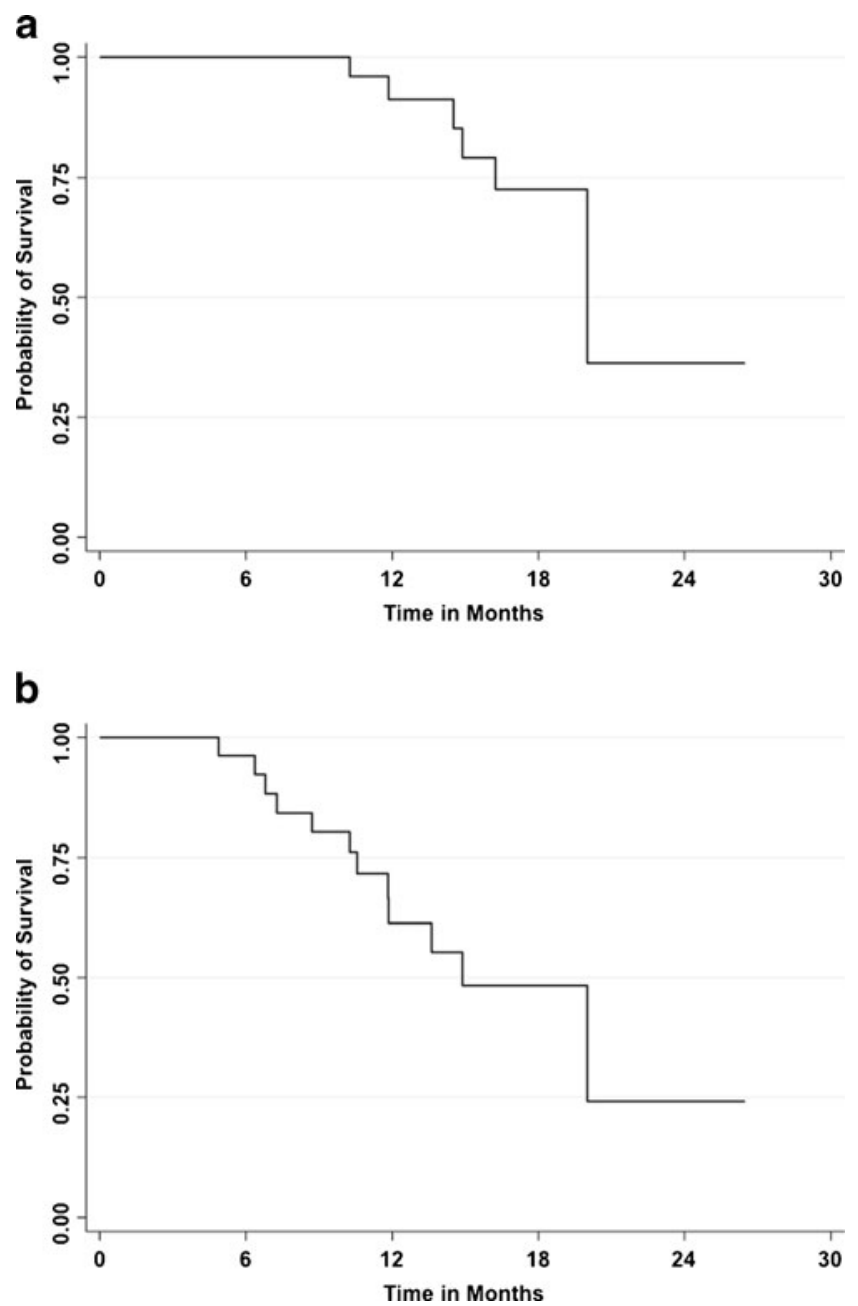

Fig. 2 Kaplan-Meier analysis of a overall survival (OS) and b progression-free survival (PFS) for patients with BRPC who completed neoadjuvant therapy

need for vessel resection. The importance of this cannot be understated since margin status is clearly associated with survival outcomes [5-7, 29, 30]. We also observed downstaging of lymph nodes despite them not being included within the RT target volumes. Of the 21 patients that were resected, 18 (60\%) were clinically node positive by EUS staging. Only five $(23.8 \%)$ patients were pathologically node positive after neoadjuvant therapy.

These data are similar to our previously published experience that included eight patients who achieved an R0 resection out of nine resected patients. Median OS and PFS were 15.6 and 10.5 months, respectively. The series has recently been updated and now includes 23 patients treated with neoadjuvant GTX-IMRT of which 14 were resected with 13 patients reported to be margin-negative and with 2 patients requiring vessel resection. Four (28.2\%) patients were pathologically node positive. In contrast, our published series of stage I and II resectable pancreatic cancer patients over a 20 -year period revealed 
that $65(47 \%)$ out of 137 patients were lymph node positive. This apparent ability of neoadjuvant regimens to downstage nodal involvement can be used as a rationale for giving neoadjuvant therapy to all resectable pancreatic cancer patients [31, 32].

The use of SBRT in pancreatic cancer was initially studied in LAPC. Koong et al. published single fraction phase I results demonstrating that up to 25 Gy was well tolerated with $100 \%$ local control at a median follow-up of 4.5 months [20]. Furthermore, the maximum tolerated dose was not reached because the primary endpoint of local control was satisfied. The subsequent phase II study reported excellent local control in 15 of 16 patients incorporating SBRT as a boost [21]. Several other studies have since reported similar results with median survival ranging from 6 to 14.3 months, local control ranging from 45 to $100 \%$, with low rates of grade 3 toxicity $[19,23,24,33$, 34]. The Danish experience, however, reported severe late mucositis, duodenal perforation, or ulceration in $42 \%$ [33]. This toxicity was likely secondary to more generous PTV expansions $(\leq 10 \mathrm{~mm})$ than other similar trials $(3-5 \mathrm{~mm})$. For instance, although the Stanford experience included tumors of similar size, their median PTV volume was $46.6 \mathrm{~cm}^{3}$ (range, $21.5-84.2 \mathrm{~cm}^{3}$ ) compared to $136 \mathrm{~cm}^{3}$ (range, $38-376 \mathrm{~cm}^{3}$ ) [34]. Nevertheless, the early collective literature suggests that SBRT is a reasonable alternative to standard fractionation RT and merits further clinical evaluation.

The ultimate goal of neoadjuvant treatment is to create a plane of resection between the tumor and adjacent vasculature in BRPC, thus increasing the likelihood of an R0 resection. With respect to RT technique, there are several potential advantages for SBRT over standard fractionation techniques. First, delivering very high ablative doses to the area of vessel abutment likely results in higher rates of tumor response and $\mathrm{R} 0$ resection as a result of increased endothelial cell apoptosis [35]. Second, excellent local control has been demonstrated with SBRT in the locally advanced setting, which is particularly beneficial for patients who remain truly unresectable after chemotherapy and SBRT. The importance of local progression in unresectable patients should not be overlooked as it can markedly affect quality of life, resulting in pain, GI obstruction, and intestinal perforation. Third, treatment over several days is clearly more convenient for the patients, especially given their limited life expectancy. Fourth, expediting RT can minimize the time that systemic chemotherapy is not given for unresectable patients.

The primary concern when designing radiation treatment plans for pancreatic lesions is meeting normal tissue constraints, especially those of the duodenum and stomach. While rates of acute toxicity in the SBRT literature for locally advanced tumors are reasonable, the incidence of late complications, especially duodenal ulceration, should not be overlooked. We observed low rates of acute RT- related complications with no grade 3 or higher adverse effects; more than $50 \%$ of all patients in this analysis reported no acute effects. While our follow-up is limited, we have no reported late toxicity with the longest follow-up being over 26 months in one patient. We anticipate that the late toxicity in this series with continued long-term follow-up will be favorable as most underwent pancreatic resection, effectively removing most, if not all, tissue located within the PTV. However, we recognize the need for close follow-up in these patients and the potential for complications, particularly gastric or duodenal, as late as 1 year after treatment [34].

Finally, the high-dose PTV in this series, treated up to $40 \mathrm{~Gy}$, was consistently at a sufficient distance from the duodenum and other mucosal structures. While the lowerdose PTV encompassed the entire pancreatic tumor volume, the prescribed dose (median, 25 Gy; range, 25-30 Gy) was within duodenal tolerance.

We recognize that a limitation of this review is its retrospective nature. We also recognize the small patient numbers. However, similar studies have not been previously published with large patient numbers [19, 20, 24]. In addition, an Eastern Cooperative Oncology Group prospective trial (E1200) evaluating neoadjuvant therapy for borderline resectable disease closed early due to poor accrual [36].

\section{Conclusion}

Neoadjuvant GTX and SBRT appear to be a well-tolerated and feasible local treatment modality for facilitating marginnegative resection in patients with BRPC. Longer follow-up is warranted to monitor potential outcomes including longterm adverse effects, but our experience to date is encouraging. Since we have found this treatment pathway to be promising, we hope to initiate a prospective trial to further evaluate this approach.

\section{References}

1. Jemal A, Siegel R, Xu J, Ward E (2010) Cancer statistics, 2010. CA Cancer J Clin 60(5):277-300. doi:10.3322/caac. 20073

2. Li D, Xie K, Wolff R, Abbruzzese JL (2004) Pancreatic cancer. Lancet 363(9414):1049-1057. doi:10.1016/S0140-6736(04)15841-8

3. Varadhachary GR, Tamm EP, Abbruzzese JL, Xiong HQ, Crane CH, Wang H, Lee JE, Pisters PW, Evans DB, Wolff RA (2006) Borderline resectable pancreatic cancer: definitions, management, and role of preoperative therapy. Ann Surg Oncol 13(8):10351046. doi:10.1245/ASO.2006.08.011

4. Abrams RA, Lowy AM, O'Reilly EM, Wolff RA, Picozzi VJ, Pisters PW (2009) Combined modality treatment of resectable and borderline resectable pancreas cancer: expert consensus statement. Ann Surg Oncol 16(7):1751-1756. doi:10.1245/ s10434-009-0413-9

5. Millikan KW, Deziel DJ, Silverstein JC, Kanjo TM, Christein JD, Doolas A, Prinz RA (1999) Prognostic factors associated with 
resectable adenocarcinoma of the head of the pancreas. Am Surg 65(7):618-623, discussion 623-614

6. Neoptolemos JP, Stocken DD, Dunn JA, Almond J, Beger HG, Pederzoli P, Bassi C, Dervenis C, Fernandez-Cruz L, Lacaine F, Buckels J, Deakin M, Adab FA, Sutton R, Imrie C, Ihse I, Tihanyi T, Olah A, Pedrazzoli S, Spooner D, Kerr DJ, Friess H, Buchler MW (2001) Influence of resection margins on survival for patients with pancreatic cancer treated by adjuvant chemoradiation and/or chemotherapy in the ESPAC-1 randomized controlled trial. Ann Surg 234(6):758-768

7. Sohn TA, Yeo CJ, Cameron JL, Koniaris L, Kaushal S, Abrams RA, Sauter PK, Coleman J, Hruban RH, Lillemoe KD (2000) Resected adenocarcinoma of the pancreas - 616 patients: results, outcomes, and prognostic indicators. J Gastrointest Surg 4(6):567-579

8. Patel M, Hoffe S, Malafa M, Hodul P, Klapman J, Centeno B, Kim J, Helm J, Valone T, Springett G (2011) Neoadjuvant GTX chemotherapy and IMRT-based chemoradiation for borderline resectable pancreatic cancer. J Surg Oncol 104(2):155-161. doi:10.1002/jso.21954

9. Mehta VK, Poen JC, Ford JM, Oberhelman HA, Vierra MA, Bastidas AJ, Fisher GA (2001) Protracted venous infusion 5-fluorouracil with concomitant radiotherapy compared with bolus 5-fluorouracil for unresectable pancreatic cancer. Am J Clin Oncol 24(2):155-159

10. Massucco P, Capussotti L, Magnino A, Sperti E, Gatti M, Muratore A, Sgotto E, Gabriele P, Aglietta M (2006) Pancreatic resections after chemoradiotherapy for locally advanced ductal adenocarcinoma: analysis of perioperative outcome and survival. Ann Surg Oncol 13(9):1201-1208. doi:10.1245/s10434-006-9032-x

11. Stokes JB, Nolan NJ, Stelow EB, Walters DM, Weiss GR, de Lange EE, Rich TA, Adams RB, Bauer TW (2011) Preoperative capecitabine and concurrent radiation for borderline resectable pancreatic cancer. Ann Surg Oncol 18(3):619-627. doi:10.1245/ s10434-010-1456-7

12. Bockbrader M, Kim E (2009) Role of intensity-modulated radiation therapy in gastrointestinal cancer. Expert Rev Anticancer Ther 9(5):637-647. doi:10.1586/era.09.16

13. Poppe MM, Narra V, Yue NJ, Zhou J, Nelson C, Jabbour SK (2011) A comparison of helical intensity-modulated radiotherapy, intensity-modulated radiotherapy, and 3D-conformal radiation therapy for pancreatic cancer. Med Dosim 36(4):351-357. doi:10.1016/j.meddos.2010.08.003

14. Yovino S, Poppe M, Jabbour S, David V, Garofalo M, Pandya N, Alexander R, Hanna N, Regine WF (2011) Intensity-modulated radiation therapy significantly improves acute gastrointestinal toxicity in pancreatic and ampullary cancers. Int J Radiat Oncol Biol Phys 79(1):158-162. doi:10.1016/j.ijrobp.2009.10.043

15. McGarry RC, Papiez L, Williams M, Whitford T, Timmerman RD (2005) Stereotactic body radiation therapy of early-stage nonsmall-cell lung carcinoma: phase I study. Int J Radiat Oncol Biol Phys 63(4):1010-1015. doi:10.1016/j.ijrobp.2005.03.073

16. Onishi H, Araki T, Shirato H, Nagata Y, Hiraoka M, Gomi K, Yamashita T, Niibe Y, Karasawa K, Hayakawa K, Takai Y, Kimura T, Hirokawa Y, Takeda A, Ouchi A, Hareyama M, Kokubo M, Hara R, Itami J, Yamada K (2004) Stereotactic hypofractionated high-dose irradiation for stage I nonsmall cell lung carcinoma: clinical outcomes in 245 subjects in a Japanese multiinstitutional study. Cancer 101(7):1623-1631. doi:10.1002/cncr.20539

17. Rusthoven KE, Kavanagh BD, Burri SH, Chen C, Cardenes H, Chidel MA, Pugh TJ, Kane M, Gaspar LE, Schefter TE (2009) Multi-institutional phase I/II trial of stereotactic body radiation therapy for lung metastases. J Clin Oncol 27(10):1579-1584. doi:10.1200/JCO.2008.19.6386

18. Scorsetti M, Mancosu P, Navarria P, Tozzi A, Castiglioni S, Clerici E, Reggiori G, Lobefalo F, Fogliata A, Cozzi L (2011) Stereotactic body radiation therapy (SBRT) for adrenal metastases: a feasibility study of advanced techniques with modulated photons and protons. Strahlenther Onkol 187(4):238-244. doi:10.1007/s00066-011-2207-9
19. Chang DT, Schellenberg D, Shen J, Kim J, Goodman KA, Fisher GA, Ford JM, Desser T, Quon A, Koong AC (2009) Stereotactic radiotherapy for unresectable adenocarcinoma of the pancreas. Cancer 115(3):665-672. doi:10.1002/cncr.24059

20. Koong AC, Le QT, Ho A, Fong B, Fisher G, Cho C, Ford J, Poen J, Gibbs IC, Mehta VK, Kee S, Trueblood W, Yang G, Bastidas JA (2004) Phase I study of stereotactic radiosurgery in patients with locally advanced pancreatic cancer. Int J Radiat Oncol Biol Phys 58(4):1017-1021. doi:10.1016/j.ijrobp.2003.11.004

21. Koong AC, Christofferson E, Le QT, Goodman KA, Ho A, Kuo T, Ford JM, Fisher GA, Greco R, Norton J, Yang GP (2005) Phase II study to assess the efficacy of conventionally fractionated radiotherapy followed by a stereotactic radiosurgery boost in patients with locally advanced pancreatic cancer. Int J Radiat Oncol Biol Phys 63(2):320-323. doi:10.1016/j.ijrobp.2005.07.002

22. Mahadevan A, Jain S, Goldstein M, Miksad R, Pleskow D, Sawhney M, Brennan D, Callery M, Vollmer C (2010) Stereotactic body radiotherapy and gemcitabine for locally advanced pancreatic cancer. Int J Radiat Oncol Biol Phys 78(3):735-742. doi:10.1016/ j.ijrobp.2009.08.046

23. Mahadevan A, Miksad R, Goldstein M, Sullivan R, Bullock A, Buchbinder E, Pleskow D, Sawhney M, Kent T, Vollmer C, Callery M (2011) Induction gemcitabine and stereotactic body radiotherapy for locally advanced nonmetastatic pancreas cancer. Int $\mathrm{J}$ Radiat Oncol Biol Phys 81(4):e615-e622. doi:10.1016/ j.ijrobp.2011.04.045

24. Polistina F, Costantin G, Casamassima F, Francescon P, Guglielmi R, Panizzoni G, Febbraro A, Ambrosino G (2010) Unresectable locally advanced pancreatic cancer: a multimodal treatment using neoadjuvant chemoradiotherapy (gemcitabine plus stereotactic radiosurgery) and subsequent surgical exploration. Ann Surg Oncol 17(8):2092-2101. doi:10.1245/s10434-010-1019-y

25. Fine RL, Fogelman DR, Schreibman SM, Desai M, Sherman W, Strauss J, Guba S, Andrade R, Chabot J (2008) The gemcitabine, docetaxel, and capecitabine (GTX) regimen for metastatic pancreatic cancer: a retrospective analysis. Canc Chemother Pharmacol 61(1):167-175. doi:10.1007/s00280-007-0473-0

26. Farma JM, Santillan AA, Melis M, Walters J, Belinc D, Chen DT, Eikman EA, Malafa M (2008) PET/CT fusion scan enhances CT staging in patients with pancreatic neoplasms. Ann Surg Oncol 15 (9):2465-2471. doi:10.1245/s10434-008-9992-0

27. Gastrointestinal Tumor Study Group (1979) Comparative therapeutic trial of radiation with or without chemotherapy in pancreatic carcinoma. Int J Radiat Oncol Biol Phys 5(9):1643-1647

28. Gastrointestinal Tumor Study Group (1988) Treatment of locally unresectable carcinoma of the pancreas: comparison of combinedmodality therapy (chemotherapy plus radiotherapy) to chemotherapy alone. J Natl Canc Inst 80(10):751-755

29. Kuhlmann KF, de Castro SM, Wesseling JG, ten Kate FJ, Offerhaus GJ, Busch OR, van Gulik TM, Obertop H, Gouma DJ (2004) Surgical treatment of pancreatic adenocarcinoma; actual survival and prognostic factors in 343 patients. Eur J Cancer 40(4):549558. doi:10.1016/j.ejca.2003.10.026

30. Pingpank JF, Hoffman JP, Ross EA, Cooper HS, Meropol NJ, Freedman G, Pinover WH, LeVoyer TE, Sasson AR, Eisenberg BL (2001) Effect of preoperative chemoradiotherapy on surgical margin status of resected adenocarcinoma of the head of the pancreas. J Gastrointest Surg 5(2):121-130

31. Heinrich S, Schafer M, Weber A, Hany TF, Bhure U, Pestalozzi BC, Clavien PA (2008) Neoadjuvant chemotherapy generates a significant tumor response in resectable pancreatic cancer without increasing morbidity: results of a prospective phase II trial. Ann Surg 248(6):1014-1022. doi:10.1097/SLA.0b013e318190a6da

32. Palmer DH, Stocken DD, Hewitt H, Markham CE, Hassan AB, Johnson PJ, Buckels JA, Bramhall SR (2007) A randomized phase 2 trial of neoadjuvant chemotherapy in resectable pancreatic cancer: 
gemcitabine alone versus gemcitabine combined with cisplatin. Ann Surg Oncol 14(7):2088-2096. doi:10.1245/s10434-007-9384-x

33. Hoyer M, Roed H, Sengelov L, Traberg A, Ohlhuis L, Pedersen J, Nellemann H, Kiil Berthelsen A, Eberholst F, Engelholm SA, von der Maase H (2005) Phase-II study on stereotactic radiotherapy of locally advanced pancreatic carcinoma. Radiother Oncol 76(1):4853. doi:10.1016/j.radonc.2004.12.022

34. Schellenberg D, Goodman KA, Lee F, Chang S, Kuo T, Ford JM, Fisher GA, Quon A, Desser TS, Norton J, Greco R, Yang GP, Koong AC (2008) Gemcitabine chemotherapy and single-fraction stereotactic body radiotherapy for locally advanced pancreatic cancer. Int J Radiat Oncol Biol Phys 72(3):678-686. doi:10.1016/j.ijrobp.2008.01.051
35. Brown KM, Siripurapu V, Davidson M, Cohen SJ, Konski A, Watson JC, Li T, Ciocca V, Cooper H, Hoffman JP (2008) Chemoradiation followed by chemotherapy before resection for borderline pancreatic adenocarcinoma. Am J Surg 195(3):318-321. doi:10.1016/j.amjsurg.2007.12.017

36. Landry J, Catalano PJ, Staley C, Harris W, Hoffman J, Talamonti $\mathrm{M}, \mathrm{Xu}$ N, Cooper H, Benson AB 3rd (2010) Randomized phase II study of gemcitabine plus radiotherapy versus gemcitabine, 5fluorouracil, and cisplatin followed by radiotherapy and 5fluorouracil for patients with locally advanced, potentially resectable pancreatic adenocarcinoma. J Surg Oncol 101(7):587-592. doi:10.1002/jso.21527 